\title{
Name index
}

Abenius 287, 289-94, 506

Abrahamsson 297

Agfors 177

Ahlgren 128-34, 136, 138-9, 142, 268, 396, $438,478,568$

Ahrsjö 234

Åkerman 226, 297

Alsholm 333

Altbäck 163, 175, 184, 209-10, 224, 244-5, 504,567

Åman 121

Ametier 123, 128, 427

Angelini 333

Arnault family 409-10, 534, 550

Åsling 108-9, 129, 298,

Asserståhl 132, 137, 139, 141-2, 148, 568

Åström 276-7, 395, 433, 437, 441, 444-5, 567

Atmer 302

Augustsson 430, 439

Bahrke 111-13, 115-17, 121, 382, 568

Barnevik 352-3, 357-60, 518, 524

Berggren (Bo) 296-8, 304, 306, 309-13, 318-25, 327-45, 347, 349-59, 416, 433, 509-11, 514-19, 523-4, 567

Berggren (Nils) 376

Bergkvist 387

Bertorp 146, 404, 409, 433, 437, 442, 567

Bexelius 191

Björkegren 225, 240-41, 399

Björnsson 140, 148, 151

Blomberg 140

Bohman 396

Bonde 305

Bonnier family/dynasty $81,197-8,201,227-33$, $238,241,397,399-400,493,533$

Bonnier (Albert) 228-31, 233

Bonnier ('Joja') 201, 228-35, 238, 241, 246, 493,503

Brandinger 121

Brändström 221, 224, 371, 377, 396, 399, 400, 402,504

Braun 239, 241, 248, 421

Browaldh (Ernfried) 87

Browaldh (Tore) 91, 161-2
Bruzelius 91

Bünsow family 69

Bystedt 326-7

Carlberg 327, 402

Carlgren 91, 157-8, 160-65, 167-73, 175-8, 180-83, 185-9, 203, 208-12, 214-16, 218-53, 257-8, 265, 280, 301-2, 304, 310, 376, 384, 395, 397-9, 403, 421-4, 426, 464, 486-8, 491-94, 497, 499-504, 524, 537-8, 550,567

Carlsson (Hans) 114-16, 118, 120-23, 128, $129,140,568$

Carlsson (Roine) 389

Carlsund 144

Cavalli-Björkman 196

Cerderlund 150-51

Chan 353, 356

Christiansson 130

Clausen 288-91, 293

C:son Bonde 196, 221

Dahlbäck 301, 305-6, 311-12, 318-20, 322, $324,327-8,335,337,341-2,350,352-4$, $356,358-60,433,509,517-19,524,567$

Dahlgren 226, 567

Dahlsten 308-9, 326-7, 383-90, 399, 409, $549-50,567$

Dahlström 197, 218

Danielsson (Bertil) 107, 118, 123, 128-30

Danielsson (Nils) 160

Dau 248

Daun 377, 384-7, 389, 398-99, 402, 409, 411-12, 430-31, 534, 539, 550, 567

Detter 138, 140

Dickson family 69

Diedrichs (Erik) 156-7, 166, 171-3, 177, 224, 504

Diedrichs (Harry) 245, 567

Dockered 128, 130, 133, 137-40, 149, 150-51, 568

Domeij 151

Douglas 199

Dunker 81

Duveblad 140

Dyrhage 210, 233-4, 236 
Edström (Gösta) 102-5, 109, 314

Edström (John Olof) 113

Ehrnrooth family/dynasty 354, 517

Ehrnrooth (Guy) 332, 354-5, 425-6, 518

Eidem 291, 300

Ekman family 97, 105

Ekman (Jan (Handelsbanken)) 398

Enander 256, 382, 399, 413, 435, 567

Engman 108-9, 116-17, 196, 218, 234, 237, $241,248,253,302,319-20$

Enström 366, 368, 370, 372, 374, 377, 381, 391

Ericson 278-81

Eriksson (Kenneth) 404

Eriksson (P-O) 424

Erkén 271

Fahlin 188, 209-11, 567

Fälldin 108

Feinsilber 422

Feltner 421, 430-31

Flick brothers/family 334-6, 416, 513, 524, 550

Flory 123

Forsgren $389,411,421,428,430$

Francke 68, 87, 288, 391, 422, 435, 567

Frölander 279, 404, 413, 417, 423, 436, 567

Gardell 146, 434-6, 438-42, 540-42, 550, 567

de Geer family 63

de Geer (Carl) 290

Geginat 334-5, 338

Giomi family 414

Göransson 366

Grafström 312, 330, 347, 567

Gripenstedt 199, 234

Grünewald 170

Gustafsson (Allan) 184

Gustafsson (Sten) 310

Gyll 436, 438, 440, 567

Gyllenhammar 300-307, 309-11, 422, 425, 509

Haegerstrand 323, 567

Hagelin 311, 328, 331-2, 341-2, 567

Häger 181

Häggkvist 163

Hägglund 123, 148, 338, 345, 351-3, 357-60, $517-19,567$

Haglund 219-20, 504

Hagman 130

Hamrefors 101-2, 112, 121-2, 568

Härmälä 354, 358-9, 519, 524

Harrison 323

Hedberg family 97, 384
Hedelius 421-2, 428, 435, 438, 567

Hedlund 102-8, 396, 475-7

Hedström family 105

Hedström (Bo S.) 109, 118-19, 121, 123, 128, 130,568

Heinzel (Alfred) 413, 430, 444-5, 535, 550

Heinzel (Wilfred) 413

Helgesson 140-41, 338, 340-51, 353-4, 356-9, $514-15,517-19,525,567$

Henrikson 196, 218, 398

Hèrnod 85, 88-9, 366-7, 464

Hessner 181

Hindemark 384

Högberg 105

Höglund 340

Huss 109

Hwass 91

Johansson (Leif) 322

Johansson (Lena) 151

Johansson (Manne) 113

Johansson (Sven) 113

Johansson (Sven-Åke) 320

Johansson-Hedberg 151

Johnson dynasty/family 81

Johnsson 329

Kempe dynasty/family $69-70,76,80-81,83$, $88,91,97,157-8,160-61,163,168,171$, $175,178,180,184,186-7,210,213-14$, 221-2, 225-7, 235, 240-46, 248, 269, 271, 280, 399, 461-4, 488, 492, 497, 503, 538

Kempe (Anders) 158, 249-50

Kempe (Bernhard) 70, 73

Kempe (Carl Jr) 158, 161, 163, 184-5, 188, 209, $211,214,221-3,225-6,234-6,239-40$, 243, 245-50, 254-7, 267, 280, 423-5, 432, $441,504,538,544,550,56$

Kempe (Carl Sr) 77, 80, 83-5, 90-91, 98, 104, $156-61,163,165,167,169,171,180,204$, 457, 463-4, 466-7, 499

Kempe (Erik) 91, 99, 156-61, 167, 204, 485

Kempe (Fanny) 91, 158, 160

Kempe (Frans Jr) 158, 189, 236-7, 242, 244-5, 249,567

Kempe (Frans Sr) 70, 76-7, 80, 83, 98-9, 158-61, 171, 184, 457, 463

Kempe (J.C.) 66, 69-70, 72, 76, 91, 158, 462-3, 466

Kempe (Margareta) 248-9

Kempe (Ragnar) 99, 158, 160-61

Kempe (Seth) 70, 77, 91, 98, 157-8, 160, 463

Kempe (Stig) 158, 160-61

Kempe (Sven) 158, 249

Kempe (Vivianne) 158, 248-50, 257 
Kempe (Wilhelm) 70, 73

Kneeland 381

Knuts 328, 331-3, 337-8, 341-2, 344, 351, 353, $356,358,567$

Köhler 248

Kreuger 80, 86-9, 92, 247, 464, 468

Lagergren 157-60

Larsson 248, 267, 326-9, 424, 567

Lejeune 164-5, 224, 226, 244, 252-3, 492-3, 503-4

Lemne 114-15

Liffner 151

Linde 109

Lindén 306

Lindqvist 109

Lindström 123, 371

Lippincott 400

Löf 85, 163, 176-8, 187-9, 209-13, 225-7, 229, 232-4, 236-9, 245, 247-8, 250-57, 264-5, $351,384,403,423-6,441,493-4,550,567$

Lund 123, 128-30, 133, 136, 138, 140

Lundberg (Fredrik) 200-201, 228, 237, 239-47, 249-58, 264-8, 270-73, 275-6, 279, 281, $351,422-6,432,441,493-7,499-504$, $537-8,550,567$

Lundberg (Lars Erik) 200

Lundberg (Per) 305

Lundin 254, 277, 279, 281-2, 567

Lundquist 194

Lundqvist 294-5

Lundström 142

Lundvall 300

Lyberg 113-15, 157, 160-62, 164, 166, 168-9, 172,183

Lyfors 108

Magnusson 140-44, 146, 150-51, 438, 550, 568

Mannerfelt 105, 199-200, 310, 382, 385, 395, 504,567

Mårtensson 248, 253, 255, 258, 567

Martin-Löf (Roland) 251-2

Martin-Löf (Rutger) 104

Martin-Löf (Sverker) 128, 146, 187, 243, 247-8, 256, 258, 275-6, 351, 389, 394, 402-4, 409-14, 416-18, 420-26, 429-33, $437-8,440-42,444-5,496,504,533-4$, $549-50,567$

Mogren 312, 339, 343, 356, 568

Mossberg 105, 172, 177, 371-7, 383, 391, 526, $529,546,549$

Nabseth 188, 209, 245, 567

Nilsson 223, 226
Norberg 382, 391, 567

Nordberg, 127

Nordenö 382, 384, 393, 395, 409, 411-12, $414-15,417-18,567$

Nordin 221, 228, 234, 239-41, 248, 393

Nordlander 113

Nordlund 170, 177, 180-81, 227, 567

Nordmark-Nilsson 130

Norlin 101, 271

Nydahl 115, 120

Öberg 135,422

Olofsson 139, 151, 568

Olsson (Curt) 307

Olsson (William) 70, 463

Önnesjö 198-201, 227, 229-31, 234-5, 237, 241, 254-7, 264-5, 268, 310, 397, 426, 490, 504

Palme (Gunnar) 139, 141-7, 150, 438, 568

Palme (Olof) 381

Palmstierna 241, 300, 302-3, 310, 312, 322, $328-9,338,425,568$

Penser 327, 402, 409, 418, 422-4, 428, 433, 435, 567

Peppler 162, 165-6, 169-70, 172, 176-7, 181, $183-4,224,504,567$

Persson (Göran) 137-8, 143, 150, 484, 568

Persson (Karl-Erik) 121, 123

Persson (Rune) 312, 318, 320, 324, 342

Persson (Tommy) 229

Petersson 148, 311, 323-5, 327, 330, 332, 335, $340-41,345,349,359,519,525,568$

Pettersson (Bengt) 264-72, 274-8, 338, 441, 495, 515, 567

Pettersson (Hans) 146, 148, 277

Pettersson (Karl-Henrik) 123, 568

Pettersson (Torbjörn) 338

Pihl 354, 383, 425

Pihlgren 383-4

Rausing (Hans) 115

Rausing (Ruben) 115-16

Redefelt 116

Rekke 140, 438

Reuterskiöld 198-200, 202, 228-9, 231, 234, $238,433,440,567$

Rietz 404, 409, 417, 423, 436-7, 567

Ros 140,568

Rosén 123

Rosengren 138-40, 143, 147, 149-51, 568

de Ruvo 380, 403-4, 413, 437

Rydbo 109-10, 113-14, 116-17

Rydh 123 
Rydin $117,177,189,243,245-8,254-58$, $264,298,337,351,355,376-82,384-92$, 395-404, 409-10, 413, 415-16, 418, 420-33, 435-6, 440-42, 444-5, 504, $528-34,537-8,549-50,567$

Rynell 101

Scharp 340

Schollander 170

Schotte 116, 118, 120, 425

Sjöberg 113

Sköld (Lars) 138, 151

Sköld (Per) 111, 113-15, 117, 382

Smurfit 129, 392, 438, 443

Söderberg dynasty/family $81,197-8,200-201$, 227-30, 232, 324, 401, 493, 533

Söderberg (Erik) 199, 202, 232

Söderberg (Lena) 151

Söderberg (Ragnar) 191, 196, 198

Söderberg (Sven) 200, 228-31, 324, 343, 356, $358,397,401,493,567$

Sprängare 123, 188, 208-14, 219, 223-6, 310, 403,567

Stahle 191, 196, 216-18, 221, 301-2

Stén 87

Stenbeck family 97,116

Stenbeck (Hugo) 106

Stenbeck (Jan) 130, 144, 334, 513, 524

Strömbeck 113

Sundberg family 92

Sundberg (Ove) 386

Sundberg (Urban) 177

Sundblad (Erik) 218, 287, 290-95, 297-8, 302, 304, 306, 308-12, 322, 348, 375, 390, 398, $506,508-9,524$

Sundblad (Gunnar) 75-6, 85, 92, 172, 189-91, $218,292,467$

Sundblad (Knut) 70, 463

Sundblad (Lars G.) 191-3, 195-6, 199, 201, $215,217-19,221,229,234,240-41,292$, $297,303,319,398,425,489,503-4,550$, 567

Sundstrom 382

Sundström 139, 568

Svedberg 248, 250, 253-4, 265, 424-25, 567

Svensson 196, 228, 235, 253, 504, 567

von Sydow 197

Synnergren 220

Tamm dynasty/family $67,71,463,465$

Tamm (Gustaf) 71

Tamm (Hugo) 71

Tamm (P.A.) 71

Tegnér 128, 130, 133, 140, 568
Thulin 382-3

Thunholm 196, 309, 319, 326-7, 389, 524

Tiveus 148

Treffenberg (Ernst) 157

Treffenberg (Ewa) 158

Trolle-Löwén 199, 234, 567

Trygger dynasty/family 91-2, 191, 195

Trygger (Carl) 92, 189, 191, 193-7, 375

af Ugglas 191, 195

Ulfsparre 84, 172

de Verdier 203

Versteegh dynasty/family $68-9,80-82,87,97$, 332,463

Versteegh (Arend) 68, 81

Versteegh (Gerhard) 81, 104, 216, 303, 381, 395,476

Wachtmeister 358

Wahlström 116, 297-8, 320, 368, 375, 377-9, 381-2, 384-8, 395-6, 530, 549-50, 567

Wahren dynasty/family $67,75,78$

Wahren (Carl) 75, 197, 467

Wahren (Ernst) 75, 467

Wall 319-20

Wallander 163, 186, 188, 194, 198, 209-10, 212-13, 215, 218, 225-7, 231-3, 238, 240-41, 245, 248, 375, 394, 397-8, 424, 504,567

Wallenberg dynasty/family/sphere $74,78,81$, $87,92,97,109,115,173,196,214-16$, 219-20, 228, 233, 250, 285-6, 288, 292, 295-6, 299-303, 305-7, 309-11, 313-14, 318-19, 324, 326-9, 334, 336-7, 340, 343, $347,352,354-5,357-9,375,386-7,389$, 395, 398-401, 461, 464-5, 467, 491-2, 499, $505-13,517-19,522-4,532-3$

Wallenberg (Jacob Jr) 286, 311, 323, 340, 344, $352-3,356,358,515,568$

Wallenberg (Jacob Sr) 92-3, 191, 194, 285-92, 294-6, 300, 305, 311, 314-15, 375, 465, $505-7,509,522-4$

Wallenberg (Marc) 286, 305, 311

Wallenberg (Marcus Jr) 286, 311, 340, 344, 352-3, 357-8, 568

Wallenberg (Marcus Sr) 92-3, 108, 173, 195, $215,219,285-9,291-3,295-8,300-302$, $305,308,310-11,314-15,319,326,334$, $387,398,416,465,492,499,502,504-9$, $513,522-4,550$

Wallenberg (Marcus the Elder) 70, 78, 92-3, 286, 314, 323, 458, 463-5

Wallenberg (Peter) 286, 293, 296, 299-300, 
304-13, 318, 320-21, 323-4, 326-9, 331-5, Westergren 333

337-8, 340-44, 347, 352, 357-8, 400, 509-11, 514-15, 522-5

Wallgren 382

Westerlund 106

Westermark-Bexelius 191, 195

Welin 248, 252-3, 424, 567

Wenner-Gren 89, 367, 464

Werthén 297

Westerberg 128, 130

Wettergren 169, 209, 248

Zetterberg 200-203, 228-32, 234, 237, 250, $256,403,422,424-6,490,567$

Zumstein 166 